Commun. Fac. Sci. Univ. Ank. Ser. A1 Math. Stat.

Volume 69, Number 1, Pages 461-472(2020)

DOI: $10.31801 /$ cfsuasmas. 544204

ISSN 1303-5991 E-ISSN 2618-6470

http://communications.science.ankara.edu.tr/index.php?series=A1

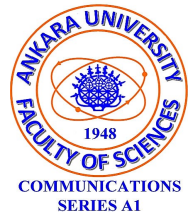

\title{
LCN-TRANSLATION SURFACES IN AFFINE 3-SPACE
}

\author{
NURAL YUKSEL, MURAT KEMAL KARACAN, AND YILMAZ TUNÇER
}

Abstract. We consider translation surfaces in Affine 3- space. Firstly, we give some results of translation surfaces whose mean and Gaussian curvatures vanish $[8,16]$. Further, we define and investigate LCN-translation surfaces with zero the mean and the Gaussian curvatures in Affine 3-space.

\section{INTRODUCTION}

A surface that arises when a curve $\alpha(u)$ is translated over another curve $\beta(v)$, is called a translation surface. A translation surface can be defined as the sum of the two generating curves $\alpha(u)$ and $\beta(v)$. Therefore, translation surfaces are made up of quadrilateral, that is, four sided, facets. Because of this property, translation surfaces are used in architecture to design and construct free-form glass roofing structures. A translation surface in an Euclidean 3 -space $\mathbb{E}^{3}$ formed by translating two curves lying in orthogonal planes is the graph of a function $z(u, v)=f(u)+g(v)$, where $f(u)$ and $g(v)$ are smooth functions on some interval of $\mathbb{R}[3,6$.

In 1835, H. F. Scherk studied translation surfaces in $\mathbb{E}^{3}$ defined as graph of the function $z(u, v)=f(u)+g(v)$ and he proved that, besides the planes, the only minimal translation surfaces are the surfaces given by

$$
z(u, v)=\frac{1}{a} \log \left|\frac{\cos (a u)}{\cos (a v)}\right|=\frac{1}{a} \log |\cos (a u)|-\frac{1}{a} \log |\cos (a v)|,
$$

where $a$ is a non-zero constant. These surfaces are now referred as Scherk's minimal surfaces [18].

In mathematics, an Affine space is a geometric structure that generalizes some of the properties of Euclidean spaces in such a way that these are independent of the concepts of distance and measure of angles, keeping only the properties related to parallelism and ratio of lengths for parallel line segments. Affine differential geometry is a type of differential geometry in which the differential invariants are

Received by the editors: March 25, 2019; Accepted: November 19, 2019.

2010 Mathematics Subject Classification. Primary 53A10; Secondary 53A15, 53A35.

Key words and phrases. Translation surfaces, affine 3-space, affine translation surfaces, LNsurfaces, LCN- translation surfaces.

(C)2020 Ankara University Communications Faculty of Sciences University of Ankara-Series A1 Mathematics and Statistics 
invariant under volume-preserving affine transformations. The basic difference between affine and Riemannian differential geometry is that in the affine case we introduce volume forms over a manifold instead of metrics.

In theory of surfaces, there are some special surfaces such as ruled surfaces, minimal surfaces, flat surfaces and surfaces of constant curvature in which differential geometers are interested. Liu described translation surfaces having constant Gaussian and mean curvature in the Euclidean and Minkowski space 12. Goemans studied weingarten translation surfaces Euclidean and Minkowski 3-spaces [6]. In the literature of affine differential geometry, translation surfaces have been also studied previously by many geometers $[8,10,11,15,16$, 17. Manhart gave a complete explicit classification of nondegenerate minimal translation surfaces in Affine space $\mathbb{R}^{3}$ [11. Magid and Vrancken showed that the curvatures must be zero and this is equivalent to one of the defining curves being planar. Also, they investigated other, natural, geometric conditions on translation surfaces. In particular, they classified those translation surfaces which are umbilical, affine spheres have trivial normal connection or null mean curvature vectors [10. Sun classified translation surface with nonzero constant mean curvature in Affine space $\mathbb{R}^{3}[15$. Fu and Hou gave a complete classification of nondegenerate affine translation surfaces with constant Gaussian curvature in $\mathbb{R}^{3}$ [ . Yang, Yu and Liu gave some classification results for nondegenerate linear Weingarten centroaffine translation surfaces in Affine space $\mathbb{R}^{3}[16$. Yanga and Fu obtained the complete classification of minimal affine translation surfaces in Affine space [17]. Andrade and Lewiner gave geometric properties of parametric or implicit surfaces, in particular the affine metric, the conormal and normal vectors, and the affine Gaussian and mean curvatures [1, 2]. Huamani studied the surfaces with zero affine mean curvature [7].

The spline surface is composed of quartic Clough-Tocher-type macro elements. Each element is capable of matching boundary data consisting of three points with associated normal vectors. The collection of the macro elements forms a $G^{1}$ continuous spline surface. Jutler and Sampoli constructed for polynomial spline surfaces with a piecewise linear field of normal vectors 9. Sampoli, Peternell and Jüttler showed that even the convolution surface of an LN-surface and any rational surface admits rational parametrization [14. Sampoli showed that for LN spline surfaces (surfaces with a linear field of normal vectors) a closed form representation is available 13 .

In this paper, we have pointed out the flat and minimal of the LCN-translation surfaces in Affine 3-space.

\section{Preliminaries}

In this section we will give some definitions of the main affine structures: the co-normal and normal vectors and the Gaussian and the mean curvatures. The Berwald-Blaschke metric is invariant for Affine transformations and also independent of system of coordinates. This metric is a quadric form. This quadratic form 
might not be positive definite (non-convex) case. Let $X: \Omega \rightarrow \mathbb{R}^{3}$ be a parametrization of a regular surface locally convex. The first affine fundamental form given by

$$
\mathbf{I}=L d u^{2}+2 M d u d v+N d v^{2}
$$

where

$$
L=\left[X_{u}, X_{v}, X_{u u}\right], \quad N=\left[X_{u}, X_{v}, X_{v v}\right], \quad M=\left[X_{u}, X_{v}, X_{u v}\right] .
$$

The Berwald-Blaschke metric or the second affine fundamental form given by

$$
h=\mathbf{I I}=E d u^{2}+2 F d u d v+G d v^{2},
$$

where

$$
E=\frac{L}{\left|L N-M^{2}\right|^{\frac{1}{4}}}, \quad G=\frac{N}{\left|L N-M^{2}\right|^{\frac{1}{4}}}, \quad F=\frac{M}{\left|L N-M^{2}\right|^{\frac{1}{4}}} .
$$

From now on, we shall assume that the surface is non-degenerate, that is, $L N-$ $M^{2} \neq 0$. Points $L N-M^{2}$ are negative, zero or positive are called hyperbolic, parabolic or elliptical, respectively [1, 2, 4, 7]. A transformation $A: \mathbb{R}^{3} \rightarrow \mathbb{R}^{3}$ is affine iff $A$ satisfies $A(u)=C(u)+v_{0}$, where $C$ is linear and $v_{0} \in \mathbb{R}^{3}$.

Orthonormality relationships are not preserved under an affine transformation $A$, therefore the Euclidean normal $\mathbf{N}^{e}$ is not an affine covariant vector. However, the direction of the Euclidean normal is covariant (if $\left\langle\mathbf{N}^{e}, X_{u}\right\rangle=0$, then $\left\langle A^{-T} \mathbf{N}^{e}, A X_{u}\right\rangle=$ 0 and similarly $X_{v},\langle$,$\rangle is Euclidean scalar product). Therefore, a covariant affine$ normal, called the affine conormal $\nu$ can be obtained by scaling the Euclidean normal vector

$$
\nu=\left|\mathbf{K}^{e}\right|^{-\frac{1}{4}} \mathbf{N}^{e}=\frac{X_{u} \wedge X_{v}}{\left[\nu, \nu_{u}, \nu_{v}\right]}=\frac{X_{u} \wedge X_{v}}{\left|L N-M^{2}\right|^{\frac{1}{4}}},
$$

where $L, N$ and $M$ are the coefficients of the first affine fundamental form, $\mathbf{K}^{e}$ and $\mathbf{N}^{e}$ are the Euclidean Gaussian curvature and the Euclidean normal vector, respectively [1, 2, 7].

By definition, it can be seen that $\nu \cdot d X=0$. Let $d= \pm\left[\nu, \nu_{u}, \nu_{v}\right]= \pm\left(L N-M^{2}\right)^{\frac{1}{4}}$, where the signal \pm depends on the point elliptical or hyperbolic. Using this notation, we have

$$
\nu=\frac{X_{u} \wedge X_{v}}{d} .
$$

Since the affine conormal is not in general a unitary vector, it is not orthogonal to its derivatives $\nu_{u}, \nu_{v}$. But since $\left[\nu, \nu_{u}, \nu_{v}\right]=d \neq 0$, those derivatives define a proper plane not orthogonal to $\nu$. A contravariant affine vector can then be obtained by looking at a vector orthogonal to that plane and would be the affine equivalent to the Euclidean normal. More precisely, the affine normal vector $\xi$ is defined locally by the relationship:

$$
\langle\nu, \xi\rangle=1, \quad\left\langle\nu_{u}, \xi\right\rangle=0, \quad\left\langle\nu_{v}, \xi\right\rangle=0
$$


The affine normal then satisfies:

$$
\left\langle\nu, \xi_{u}\right\rangle=\left\langle\nu, \xi_{v}\right\rangle=0
$$

This last relation shows that a local basis for the embedding space $\mathbb{R}^{3}$ at a point $p$ of the surface can be obtained by $\left[X_{u}, X_{v}, \xi\right]$. This allows to define affine structures from Cartan's moving frames theory. Denote by $\xi=\left[\xi_{1}, \xi_{2}, \xi_{3}\right]$ the affine normal vector. Thus we have

$$
\xi=\frac{\nu_{u} \wedge \nu_{v}}{\left[\nu, \nu_{u}, \nu_{v}\right]}=\frac{\nu_{u} \wedge \nu_{v}}{d}
$$

or

$$
\xi=\frac{1}{2} \frac{\left|L N-M^{2}\right|^{\frac{1}{4}}}{\sqrt{L N-M^{2}}}\left[\frac{\partial}{\partial u}\left(\frac{N X_{u}-M X_{v}}{\sqrt{L N-M^{2}}}\right)+\frac{\partial}{\partial v}\left(\frac{L X_{v}-M X_{u}}{\sqrt{L N-M^{2}}}\right)\right]
$$

or

$$
\xi=\frac{1}{2} \frac{1}{\sqrt{E G-F^{2}}}\left[\frac{\partial}{\partial u}\left(\frac{G X_{u}-F X_{v}}{\sqrt{E G-F^{2}}}\right)+\frac{\partial}{\partial v}\left(\frac{E X_{v}-F X_{u}}{\sqrt{E G-F^{2}}}\right)\right],
$$

[1, 2, 7].

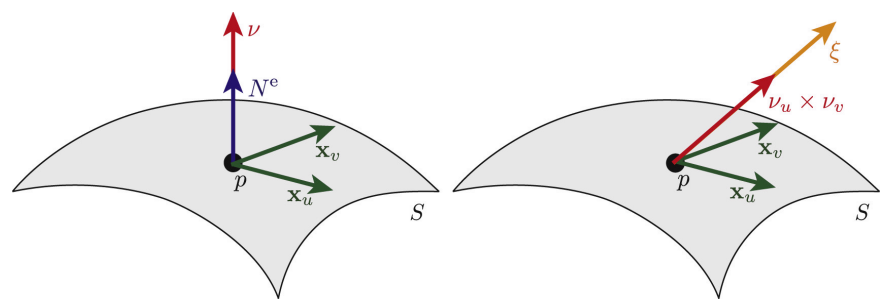

Figure 1

Observe that, the affine normal vector does not belong to the tangent plane to the surfaces $S$. The curvatures describe the variation of the normal vector. We know that $\nu . \xi_{u}=0, \nu . \xi_{v}=0$. That is , the derivatives $\xi_{u}$ and $\xi_{v}$ are orthogonal to $\nu$. In particular $\xi_{u}$ and $\xi_{v} \in T_{p} S$. Therefore, we can define the shape operator $S$ as follows

$$
S: T_{p} S \rightarrow T_{p} S
$$

given by $S_{p}(v)=-D_{v} \xi$. Since $\xi_{u}$ and $\xi_{v}$ are tangents to the surface, we have that there are functions

$$
b_{i j}: \Omega \rightarrow \mathbb{R}, i, j=1,2,
$$

such that

$$
\begin{aligned}
& \xi_{u}=b_{11} X_{u}+b_{12} X_{v}, \\
& \xi_{v}=b_{21} X_{u}+b_{22} X_{v},
\end{aligned}
$$

where

$$
b_{11}=\frac{\left[\xi_{u}, X_{v}, \xi\right]}{d},
$$




$$
\begin{aligned}
& b_{12}=\frac{\left[\xi_{v}, X_{v}, \xi\right]}{d}, \\
& b_{11}=\frac{\left[X_{u}, \xi_{u}, \xi\right]}{d}, \\
& b_{11}=\frac{\left[X_{u}, \xi_{v}, \xi\right]}{d} .
\end{aligned}
$$

This shows that in the basis $\left\{X_{u}, X_{v}\right\}$, the Shape Operator $S_{p}(v)=D_{v} \xi$ is given by the matrix $B=\left(b_{i j}\right), i, j=1,2$. Notice that this matrix is not necessarily symmetric [1, 2, 7].

Definition 1. The coefficients $b_{i j}$ form a matrix $B=\left(b_{i j}\right)$, whose determinant and the half of the trace are the Gaussian and the mean curvatures, respectively. Hence, we have

$$
\begin{aligned}
\mathbf{K} & =\operatorname{det} B=b_{11} b_{22}-b_{12} b_{21}, \\
\mathbf{H} & =\frac{1}{2} \operatorname{tr} B=\frac{b_{11}+b_{22}}{2}
\end{aligned}
$$

[1, 2, 7.

Consider a surface $X^{*}(u, v)$ in Affine 3-space. This surface is said to be an LCN (linear conormal)-surface, if its conormal vectors admit a linear representation of the form

$$
\nu^{*}=\vec{a} u+\vec{b} v+\vec{c}
$$

with certain constant coefficient vectors $\vec{a}, \vec{b}, \vec{c} \in \mathbb{R}^{3}$. More precisely, it satisfies the equations

$$
\left\langle X_{u}^{*}, \nu^{*}\right\rangle=\left\langle X_{v}^{*}, \nu^{*}\right\rangle=0
$$

We assume that the three vectors $\vec{a}, \vec{b}, \vec{c}$ are linearly independent. Without loss of generality we may then assume that

$$
\vec{a}=(1,0,0), \vec{b}=(0,1,0), \vec{c}=(0,0,1),
$$

i.e., $\nu^{*}=(u, v, 1)$. The tangent planes of an $\mathrm{LCN}$ surface have the equations

$$
T(u, v): z(u, v)+u x+v y+z=0,
$$

where $z(u, v)$ is a polynomial or rational function, in case of a polynomial or rational LCN surface, respectively. On the other hand, given a system of tangent planes of the form (11) with a polynomial or rational function $z(u, v)$. The envelope surface $X^{*}=(x, y, z)$ satisfies the equations

$$
\begin{aligned}
T(u, v) & : \quad z(u, v)+u x+v y+z=0 \\
T_{u}(u, v) & : \quad z_{u}+x=0 \\
T_{v}(u, v) & : \quad z_{v}+y=0
\end{aligned}
$$


and the conormal vector evaluates to

$$
\nu^{*}=\left(\frac{z_{u u} z_{v v}-\left(z_{u v}\right)^{2}}{d^{*}}\right)(u, v, 1) .
$$

Thus the envelope surface given by

$$
X^{*}(u, v)=\left(-z_{u},-z_{v},-z+u z_{u}+v z_{v}\right) .
$$

[13, 14 .

\section{LCN-Translation Surfaces in Affine 3-Space}

In this chapter, we define the LCN-translation surfaces in Affine 3-space. Consider a surface in as a the graph of a function $z=r(u, v)$ of two variables, which is itself the sum of two functions $f$ and $g$ of one variable. Here, we restrict our topic to regular surfaces $X$. Thus, we can express in open form as

$$
X: \quad z=f(u)+g(v) .
$$

A surface $S$ defined as the sum of two affine space curves $\alpha(u)=(u, 0, f(u))$ and $\beta(v)=(0, v, g(v))$ is called a translation surface in Affine 3-space. So, a translation surface is defined by a patch

$$
X(u, v)=(u, v, f(u)+g(v)) .
$$

The coefficients of the first affine fundamental form of the translation surface given by

$$
\begin{aligned}
& L=f^{\prime \prime}(u), N=g^{\prime \prime}(v), M=0 \\
& d=\left(L N-M^{2}\right)^{\frac{1}{4}}=\left(f^{\prime \prime} g^{\prime \prime}\right)^{\frac{1}{4}} .
\end{aligned}
$$

Hence the coefficients of the Berwald-Blaschke metric of the translation surface or the coefficients of the second affine fundamental form of the translation surface are given by

$$
E=\frac{f^{\prime \prime}}{\left(f^{\prime \prime} g^{\prime \prime}\right)^{\frac{1}{4}}}, G=\frac{g^{\prime \prime}}{\left(f^{\prime \prime} g^{\prime \prime}\right)^{\frac{1}{4}}}, F=0 .
$$

We suppose that the Berwald-Blaschke metric is non-degenerate: $d \neq 0$. Thus, we have the affine conormal and normal vectors are given by

$$
\begin{gathered}
\nu=\left(\begin{array}{c}
\left.f^{\prime}-\frac{g^{\prime}}{\left(f^{\prime \prime} g^{\prime \prime}\right)^{\frac{1}{4}}},-\frac{1}{\left(f^{\prime \prime} g^{\prime \prime}\right)^{\frac{1}{4}}}, \frac{1}{\left(f^{\prime \prime} g^{\prime \prime}\right)^{\frac{1}{4}}}\right) . \\
-\frac{f^{\prime \prime \prime}\left(f^{\prime \prime} g^{\prime \prime}\right)^{\frac{1}{4}}}{4 f^{\prime \prime 2}}, \\
-\frac{g^{\prime \prime \prime}\left(f^{\prime \prime} g^{\prime \prime}\right)^{\frac{1}{4}}}{4{g^{\prime \prime}}^{2}}, \\
\frac{\left(f^{\prime \prime} g^{\prime \prime}\right)^{\frac{1}{4}}\left(-f^{\prime} g^{\prime \prime 2}{f^{\prime \prime \prime}+f^{\prime 2}}^{2}\left(4 g^{\prime \prime 2}-g^{\prime} g^{\prime \prime \prime}\right)\right)}{4 f^{\prime \prime 2} g^{\prime \prime^{2}}}
\end{array}\right) .
\end{gathered}
$$


respectively.

Proposition 2. Let $S$ be a translation surface with non-degenerate in Affine 3space. Then the Gaussian and the mean curvatures of $S$ can be given by

$$
\begin{aligned}
& \mathbf{K}=\frac{f^{\prime \prime \prime}{ }^{2}\left(12 g^{\prime \prime \prime}{ }^{2}-7 g^{\prime \prime} g^{(4)}\right)+f^{\prime \prime} f^{(4)}\left(-7 g^{\prime \prime \prime^{2}}+4 g^{\prime \prime} g^{(4)}\right)}{64\left(f^{\prime \prime} g^{\prime \prime}\right)^{\frac{5}{2}}}, \\
& \mathbf{H}=\left(\frac{\left(f^{\prime \prime} g^{\prime \prime}\right)^{\frac{1}{4}}\left(7 f^{\prime \prime \prime} \prime^{2}-4 f^{\prime \prime} f^{(4)}\right)}{32 f^{\prime \prime^{3}}}+\frac{\left(f^{\prime \prime} g^{\prime \prime}\right)^{\frac{1}{4}}\left(7 g^{\prime \prime \prime^{2}}-4 g^{\prime \prime} g^{(4)}\right)}{32 g^{\prime 3^{3}}}\right),
\end{aligned}
$$

respectively [10, 11, 15].

In [8, 15], Fu, Hou and Sun classified vanishing Gaussian curvature and minimal translation surfaces in the Affine 3-space, they proved the following theorems:

Theorem 3. Let $S$ be a nondegenerate affine translation surface in $\mathbb{R}^{3}$ with vanishing Gaussian curvature. Then $S$ is affinely equivalent to one of the graph of the following functions

$$
\begin{aligned}
z & =u^{2}+g(v), \\
z & =e^{u}+v^{\frac{1}{2}} \\
z & =u \ln u \pm v \ln v \\
z & =\ln u \pm \ln v \\
z & =u^{\frac{3-2 \lambda}{\lambda-1}} \pm v^{\frac{3-2 \lambda}{5-3 \lambda}}
\end{aligned}
$$

where $g(v)$ is an arbitrary function and $\lambda$ is a constant satisfying $\lambda \neq 1,2, \frac{3}{2}, \frac{5}{3}[8$.

Theorem 4. Let $S$ be a nondegenerate affine minimal translation surface in $\mathbb{R}^{3}$. Then $S$ is one of the graph of the following functions under affine transformations:

$$
\begin{aligned}
z & =u^{2} \pm v^{2}, \\
z & =u^{\frac{2}{3}} \pm v^{\frac{2}{3}}, \\
z & =u^{2} \pm v^{\frac{2}{3}}
\end{aligned}
$$

or

$$
\begin{aligned}
& z=\ln u-\ln v \\
& z= \pm \ln u \pm(1+\cosh t), \quad t+\sinh t=v \\
& z= \pm \ln u \pm(1-\cos t), \quad t-\sin t=v \\
& z= \pm(1+\cosh t) \pm(1+\cosh s), \quad t+\sinh t=u, s+\sinh s=v \\
& z= \pm(1+\cosh t) \pm(1-\cos s), \quad t+\sinh t=u, s-\sinh s=v \\
& z= \pm(1-\cos t) \pm(1-\cos s), \quad t-\sin t=u, s-\sin s=v
\end{aligned}
$$

[15. 
So, using (11) and (13), we can define the LCN- translation surfaces defined by as

$$
X^{*}(u, v)=\left(-f^{\prime}(u),-g^{\prime}(v), u f^{\prime}(u)+v g^{\prime}(v)-f(u)-g(v)\right) .
$$

A basis for the tangent vectors is given by

$$
\begin{aligned}
& X_{u}^{*}=\left(-f^{\prime \prime}, 0, u f^{\prime \prime}\right), \\
& X_{v}^{*}=\left(0,-g^{\prime \prime}, v g^{\prime \prime}\right) .
\end{aligned}
$$

The second partial derivatives of $X^{*}(u, v)$ are given by

$$
\begin{aligned}
X_{u u}^{*} & =\left(-f^{\prime \prime \prime}, 0, f^{\prime \prime}+u f^{\prime \prime \prime}\right), \\
X_{u v}^{*} & =(0,0,0) \\
X_{v v}^{*} & =\left(0,-g^{\prime \prime \prime}, g^{\prime \prime \prime}+v g^{\prime \prime \prime}\right) .
\end{aligned}
$$

The coefficients of the first affine fundamental form of the translation surface given by

$$
\begin{aligned}
L^{*} & =f^{\prime \prime^{2}} g^{\prime \prime}, N^{*}=f^{\prime \prime} g^{\prime \prime^{2}}, M^{*}=0, \\
d^{*} & =\left(L^{*} N^{*}-M^{*^{2}}\right)^{\frac{1}{4}}=\left(f^{\prime \prime^{3}} g^{\prime^{3}}\right)^{\frac{1}{4}} .
\end{aligned}
$$

Hence the coefficients of the Berwald-Blaschke metric of the translation surface or the coefficients of the second affine fundamental form of the translation surface are given by

$$
E^{*}=\frac{f^{\prime \prime^{2}} g^{\prime \prime}}{\left(f^{\prime \prime^{3}} g^{\prime \prime^{3}}\right)^{\frac{1}{4}}}, G^{*}=\frac{f^{\prime \prime} g^{\prime \prime^{2}}}{\left(f^{\prime \prime^{3}} g^{\prime \prime^{3}}\right)^{\frac{1}{4}}}, F^{*}=0 .
$$

We suppose that the Berwald-Blaschke metric is non-degenerate: $d^{*} \neq 0$. Geometrically $d^{*}>0$ means that the Euclidean Gaussian curvature does not vanish, i.e. the LCN translation surface is strongly convex. The affine conormal field of the LCN translation surface given by

$$
\nu^{*}=\frac{f^{\prime \prime} g^{\prime \prime}}{\left(f^{\prime \prime 3} g^{\prime \prime^{3}}\right)^{\frac{1}{4}}}(u, v, 1) .
$$

Thus, we have the affine normal vector

$$
\xi^{*}=\left(\begin{array}{c}
-\frac{f^{\prime \prime} f^{\prime \prime \prime} g^{\prime \prime 2}}{4\left(f^{\prime \prime 3} g^{\prime \prime 3}\right)^{3}}, \\
-\frac{{f^{\prime \prime}}^{2} g^{\prime \prime} g^{\prime \prime \prime}}{4\left(f^{\prime \prime 3} g^{\prime \prime 3}\right)^{\frac{3}{4}}}, \\
\frac{f^{\prime \prime} g^{\prime \prime}\left(u f^{\prime \prime \prime} g^{\prime \prime}+f^{\prime \prime}\left(4 g^{\prime \prime}-v g^{\prime \prime \prime}\right)\right)}{4\left(f^{\prime \prime} g^{\prime \prime \prime}\right)^{\frac{3}{4}}}
\end{array}\right) .
$$


Consequently, the coefficients $b_{i j}^{*}$ form a matrix $B^{*}=\left[b_{i j}^{*}\right]$ are given by

$$
\begin{aligned}
& b_{11}^{*}=\frac{\left(f^{\prime \prime^{3}} g^{\prime \prime^{3}}\right)^{\frac{1}{4}}\left(-5 f^{\prime \prime \prime^{2}}+4 f^{\prime \prime} f^{(4)}\right)}{16 f^{\prime \prime^{4}} g^{\prime \prime}}, b_{12}^{*}=-\frac{f^{\prime \prime \prime} g^{\prime \prime} g^{\prime \prime \prime}}{16\left(f^{\prime \prime} g^{\prime \prime^{3}}\right)^{\frac{3}{4}}}, \\
& b_{21}^{*}=-\frac{f^{\prime \prime} f^{\prime \prime \prime} g^{\prime \prime \prime}}{16\left(f^{\prime \prime}{ }^{\prime \prime} g^{\prime \prime}\right)^{\frac{3}{4}}}, b_{22}^{*}=\frac{\left(f^{\prime \prime^{3}} g^{\prime \prime^{3}}\right)^{\frac{1}{4}}\left(-5 g^{\prime \prime \prime}+4 g^{\prime \prime} g^{(4)}\right)}{16 f^{\prime \prime} g^{\prime \prime^{4}}} .
\end{aligned}
$$

Proposition 5. Let $S^{*}$ be a $L C N$-translation surface with non-degenerate in Affine 3-space. Then the Gaussian and the mean curvatures of $S^{*}$ can be given by

$$
\begin{aligned}
K^{*} & =\frac{f^{\prime \prime} g^{\prime \prime}\left(f^{\prime \prime \prime}{ }^{2}\left(6 g^{\prime \prime \prime}{ }^{2}-5 g^{\prime \prime} g^{(4)}\right)+f^{\prime \prime} f^{(4)}\left(-5 g^{\prime \prime \prime^{2}}+4 g^{\prime \prime} g^{(4)}\right)\right)}{64\left(f^{\prime \prime^{3}} g^{\prime \prime^{3}}\right)^{\frac{3}{2}}} \\
H^{*} & =\frac{1}{2}\left(\frac{\left(f^{\prime \prime^{3}} g^{\prime \prime^{3}}\right)^{\frac{1}{4}}\left(-5 f^{\prime \prime \prime^{2}}+4 f^{\prime \prime} f^{(4)}\right)}{16 f^{\prime \prime^{4}} g^{\prime \prime}}+\frac{\left(f^{\prime \prime^{3}} g^{\prime \prime^{3}}\right)^{\frac{1}{4}}\left(-5 g^{\prime \prime \prime}{ }^{2}+4 g^{\prime \prime} g^{(4)}\right)}{16 f^{\prime \prime} g^{\prime \prime^{4}}}\right)
\end{aligned}
$$

where $f^{\prime \prime} \neq 0, g^{\prime \prime} \neq 0$, respectively.

We suppose that the LCN-translation surface with non-degenerate given by 29 has zero the Gaussian curvature. Then we obtain

$$
f^{\prime \prime \prime}{ }^{2}\left(6 g^{\prime \prime \prime}{ }^{2}-5 g^{\prime \prime} g^{(4)}\right)+f^{\prime \prime} f^{(4)}\left(-5 g^{\prime \prime \prime \prime^{2}}+4 g^{\prime \prime} g^{(4)}\right)=0
$$

Here $u$ and $v$ are independent variables, so each side of $(30)$ is equal to a constant $p \in \mathbb{R} \backslash\{0\}$. Hence, the equation 30$\}$ is reduced to

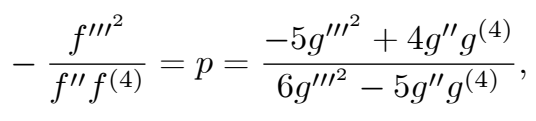

where $f^{(4)} \neq 0, g^{(4)} \neq 0$. By solving 31 , we get

$$
\begin{aligned}
& f(u)=c_{1}+c_{2} u-\frac{c_{3}\left(u+p u-p c_{4}\right)^{2+\frac{p}{1+p}}}{(1+2 p)(2+3 p)}, \\
& g(v)=c_{5}+c_{6} v-\frac{c_{7}\left((1+p) v+c_{8}(4+5 p)\right)^{-\frac{2+3 p}{1+p}}}{(2+3 p)(3+4 p)},
\end{aligned}
$$


for some constants $c_{i} \in \mathbb{R}$ and $p \neq\left\{-1,-\frac{1}{2},-\frac{2}{3},-\frac{3}{4}\right\}$. We draw it as in Figure 2 .

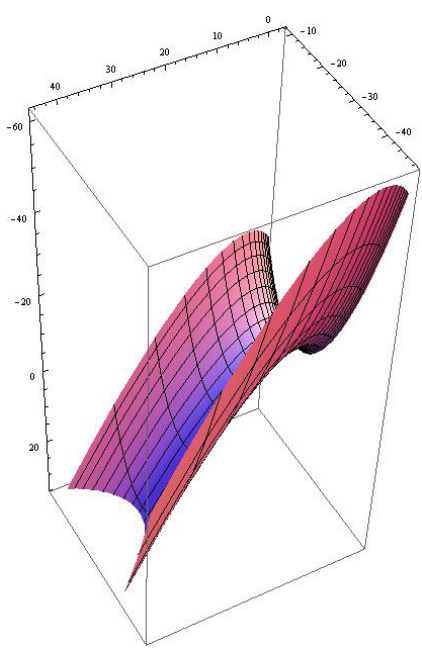

Figure 2

Theorem 6. Let $S^{*}$ be a $L C N$-translation surface with non-degenerate in Affine 3-space. If $S^{*}$ has zero Gaussian curvature or affine flat then $S^{*}$ is parametrized as 20) with (32).

We assume that $S^{*}$ is affine minimal. Hence, the mean curvature is zero if and only if

$$
\frac{\left(f^{\prime \prime^{3}} g^{\prime \prime^{3}}\right)^{\frac{1}{4}}\left(-5 f^{\prime \prime \prime^{2}}+4 f^{\prime \prime} f^{(4)}\right)}{16 f^{\prime \prime^{4}} g^{\prime \prime}}+\frac{\left(f^{\prime \prime^{3}} g^{\prime \prime^{3}}\right)^{\frac{1}{4}}\left(-5 g^{\prime \prime \prime^{2}}+4 g^{\prime \prime} g^{(4)}\right)}{16 f^{\prime \prime} g^{\prime \prime^{4}}}=0 .
$$

Then, the minimality condition (33) can be separated for the variables

$$
\frac{\left(-5 f^{\prime \prime \prime}{ }^{2}+4 f^{\prime \prime} f^{(4)}\right)}{f^{\prime \prime^{3}}}=-\frac{\left(-5 g^{\prime \prime \prime}{ }^{2}+4 g^{\prime \prime} g^{(4)}\right)}{g^{\prime \prime^{3}}},
$$

which implies there exists a constant $p \in \mathbb{R} \backslash\{0\}$ such that

$$
\frac{\left(-5 f^{\prime \prime \prime^{2}}+4 f^{\prime \prime} f^{(4)}\right)}{f^{\prime \prime^{3}}}=p=-\frac{\left(-5 g^{\prime \prime \prime^{2}}+4 g^{\prime \prime} g^{(4)}\right)}{g^{\prime \prime^{3}}},
$$

where $f^{\prime \prime} \neq 0, g^{\prime \prime} \neq 0$. Solving this equation for $f$ and $g$, we get

$$
f(u)=c_{1}+c_{2} u-\frac{2 c_{3}\left(c_{4}+u\right) \arctan h\left(\frac{c_{3}\left(c_{4}+u\right)}{4 \sqrt{p}}\right)}{p^{\frac{3}{2}}},
$$




$$
g(v)=c_{5}+c_{6} v-\frac{2 c_{7}\left(c_{8}+v\right) \arctan h\left(\frac{c_{7}\left(c_{8}+v\right)}{4 \sqrt{p}}\right)}{p^{\frac{3}{2}}},
$$

where $c_{i} \in \mathbb{R}$ and $p \in \mathbb{R} \backslash\{0\}$. We draw it as in Figure 3 .

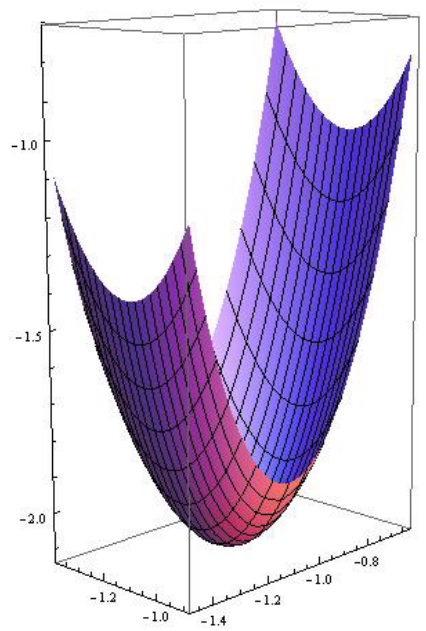

Figure 3

Thus we have following theorem.

Theorem 7. A LCN-translation surface with non-degenerate $S^{*}$ is affine minimal in Affine 3-space if and only if it is a part of the surface (20) with (36).

\section{REFERENCES}

[1] Andrade, M. Calculus of Affine Structures and Applications for Isosurfaces (in Portuguese), PhD dissertation, Rio de Janeiro, August 2011.

[2] Andrade, M. and Lewiner, T. Affine-invariant Curvature Estimators for Implicit Surfaces, Computer Aided Geometric Design 29 (2012),162-173.

[3] Arslan, K., Bayram, B., Bulca, B. and Ozturk, G. On translation surfaces in 4-dimensional Euclidean space, Acta Et Commentationes Universitatis Tartuensis De Mathematica, 20(2) (2016),123-133.

[4] Blaschke, W. Vorlesungen über Differentialgeometrie II, Springer, Berlin, 1923.L. Verstraelen, L. Vrancken, Affine variation formulas and affine minimal surfaces, Michigan Math. J., 36 (1989), 77-93.

[5] Bulca, B. On generalized LN-Surfaces in $\mathbb{E}^{4}$, Mathematical Sciences And Applications ENotes, 1(2) (2013), 35-41.

[6] Goemans, W. Surfaces in three-dimensional Euclidean and Minkowski space, in particular a study of Weingarten surfaces, PhD. Dissertation, September 2010.

[7] Huamani, E. F. C. Affine Minimal Surfaces with Singularities, Masters dissertation,Rio de Janeiro,September 2017.

[8] Fu, Y. and Hou, Z.H. Affine Translation Surfaces with Constant Gauss Curvatures, Kyungpook Math. J., 50 (2010), 337-343. 
[9] Juttler, B. and Sampoli, M.L. Hermite interpolation by piecewise polynomial surfaces with rational offsets, Comp. Aided Geom. Design, 17 (2000), 361-385.

[10] Magid, M. and Vrancken, L. Affine Translation Surfaces, Results Math., 35 (1999),134-144.

[11] Manhart, F. Die Affinminimalrückungsflächen, Arch. Math., 44 (1985), 547-556.

[12] Liu, H.L. Translation Surfaces with Constant Mean Curvature in 3-dimensional spaces, J.Geom., 64 (1999), 141-149.

[13] Sampoli, M.L. Computing the convolution and the Minkowski sum of surfaces, Proceedings of the 21st Spring Conference on Computer Graphics, Budmerice,Slovakia, May 12-14, (2005),111-117

[14] Sampoli, M. L., Peternell, M. and Jüttler, B. Rational surfaces with linear normals and their convolutions with rational surfaces, Comp. Aided Geom. Design, 23 (2006), 179-192

[15] Sun, H. On affine translation surfaces of constant mean curvature, Kumamoto J. Math., 13 (2000) $49-57$.

[16] Yang, Y., Yu, Y.H. and Liu, H.L. Linear Weingarten centroaffine translation surfaces in $\mathbb{R}^{3}$, J. Math. Anal. Appl., 375 (2011), 458-466.

[17] Yanga, D.Fu, Y.,On affine translation surfaces in affine space, J. Math. Anal. Appl., 440 (2016), 437-450.

[18] Yoon, D.W. Some Classification of Translation surfaces in Galilean 3-space, Int. Journal of Math. Analysis, 6(28) (2012), 1355-1361.

Current address: Nural Yuksel: Erciyes University, Faculty of Sciences, Department of Mathematics, 38030- Melikgazi / KAYSERI

E-mail address: yukseln@erciyes.edu.tr

ORCID Address: https://orcid.org/0000-0003-3360-5148

Current address: Murat Kemal Karacan: Usak University, Faculty of Sciences and Arts, Department of Mathematics,1 Eylul Campus, 64200,Usak-TURKEY.

E-mail address: murat.karacan@usak.edu.tr

ORCID Address: https://orcid.org/0000-0002-2832-9444

Current address: Yılmaz Tunçer: Usak University, Faculty of Sciences and Arts, Department of Mathematics,1 Eylul Campus, 64200,Usak-TURKEY.

E-mail address: yilmaz.tuncer@usak.edu.tr

ORCID Address: https://orcid.org/0000-0002-2398-866X 